\title{
Ultrasound-modulated optical tomography of biological tissue by use of contrast of laser speckles
}

\author{
Jun Li, Geng Ku, and Lihong V. Wang
}

\begin{abstract}
Ultrasound-modulated optical tomography based on the measurement of laser-speckle contrast was investigated. An ultrasonic beam was focused into a biological-tissue sample to modulate the laser light passing through the ultrasonic column inside the tissue. The contrast of the speckle pattern formed by the transmitted light was found to depend on the ultrasonic modulation and could be used for imaging. Variation in the speckle contrast reflected optical inhomogeneity in the tissue. With this technique, two-dimensional images of biological-tissue samples of as much as $25 \mathrm{~mm}$ thick were successfully obtained with a low-power laser. The technique was experimentally compared with speckle-contrastbased, purely optical imaging and with parallel-detection imaging techniques, and the advantages over each were demonstrated. (C) 2002 Optical Society of America
\end{abstract}

OCIS codes: $170.3880,120.6150,110.7050,110.7170$.

\section{Introduction}

Strong optical scattering is a significant problem in the optical imaging of biological tissues. Because ultrasonic waves are scattered less in biological tissues than are optical waves, ultrasonic waves can provide more accurate localization information. Consequently, hybrid techniques that combine optical and ultrasonic waves and that take advantage of both optical contrast and ultrasonic resolution have been explored. Ultrasound-modulated optical imaging is one of these hybrid techniques and has been studied by several research groups. Marks et al. ${ }^{1}$ investigated the combination of pulsed ultrasound and laser light and detected ultrasound-modulated optical signals in a homogeneous turbid medium. Wang et al. ${ }^{2}$ and Wang and $\mathrm{Zhao}^{3}$ developed ultrasound-modulated optical tomography that combined continuous-wave ultrasound and laser irradiation and obtained images in tissue-simulating turbid media. Leutz and Maret $^{4}$ studied the ultrasonic modulation of multiply scattered light by measuring the autocorrelation functions and the light intensity

The authors are with the Optical Imaging Laboratory, Biomedical Engineering Program, Texas A \& M University, 3120 Texas A \& M University, College Station, Texas 77843-3120. L. V. Wang's e-mail address lwang@tamu.edu.

Received 14 December 2001; revised manuscript received 18 April 2002.

0003-6935/02/286030-06\$15.00/0

(C) 2002 Optical Society of America spectra. Wang and $\mathrm{Ku}^{5}$ employed a frequencyswept technique to achieve controllable spatial resolution along the ultrasonic axis. Leveque et al. ${ }^{6}$ developed a parallel speckle detection in which a CCD camera worked as a detector array and the signal-to-noise ratio (SNR) was improved by averaging of the signals from all of the CCD pixels. They obtained one-dimensional (1D) images of biological tissue. Yao and Wang ${ }^{7}$ subsequently obtained twodimensional (2D) images of multiple objects buried in biological tissue. Yao et al. ${ }^{8}$ further developed the technique by combining the parallel detection and the frequency-swept techniques, and they obtained $2 \mathrm{D}$ images of biological tissues in which one dimension was along the ultrasonic axis. Using parallel detection, Leveque-Fort ${ }^{9}$ obtained three-dimensional images in biological tissue. In addition to imaging in transmission configurations, imaging in reflection configurations was studied by Lev et al., ${ }^{10}$ Granot et $a l .,{ }^{11}$ and Leveque et $a l .{ }^{12}$ Comprehensive theoretical models describing the mechanism of ultrasonic modulation of multiply scattered light, which includes both analytic and Monte Carlo models, have been developed by Wang ${ }^{13,14}$ recently.

All of the imaging techniques applied in ultrasound-modulated optical tomography are based on the detection of ultrasound-tagged light. Among these techniques, parallel detection ${ }^{6-9,12}$ is the most efficient technique. However, in parallel detection, because four acquisitions are needed to obtain imaging information of a location at the ultrasonic column and each acquisition must collect sufficient photons 


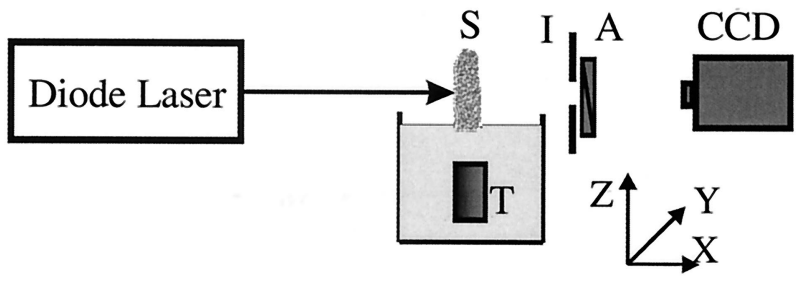

Fig. 1. Experimental setup: S, sample; I, iris; A, analyzer; T, transducer.

to maintain enough SNR, the long acquisition time involved may lead to speckle decorrelation.

In this paper, we present our investigation of an imaging method based on the detection of laserspeckle contrast. In purely optical imaging, Thompson et al., ${ }^{15}$ McKinney et al., ${ }^{16}$ and Naulleau et al. ${ }^{17}$ have applied speckle contrast in the imaging of scattering media in transmission geometries, and in the biomedical field, speckle contrast has been used in the optical characterization of surface roughness ${ }^{18}$ and in the detection of skin disease in lightly scattering thin samples. ${ }^{19}$ Jacques and Kirkpatrick ${ }^{20}$ used speckle contrast in the acoustically modulated speckle imaging of biological tissue surfaces. Their experiment was somewhat similar to ours in that acoustic waves were applied and speckle contrast was measured. But they did the experiment using reflection geometry, at lower acoustic frequencies $(0-30$ $\mathrm{Hz}$ ), and the greatest difference between our experiment was that the acoustic modulation was not used for improving spatial resolution. The purpose of their experiment was to discriminate superficial tissue layers, which had different mechanical properties and thus had different laser-speckle patterns in response to mechanical driving forces from the acoustic waves. The technique we propose here is intended to image objects buried deeply in biological tissues by taking advantage of ultrasonic resolution. It is a case of transmission geometry, and the image contrast is based on the optical properties of the tissues.

Our experiments showed that when ultrasound acted on the tissues, the contrast of the speckle pattern formed by the transmitted light decreased. The ultrasound-dependent speckle contrast could be used for tissue imaging. By measuring the variation of the speckle contrast with the location of ultrasonic column, one could detect optical inhomogeneities inside the tissue even if the tissue is acoustically homogeneous. Using this technique, we obtained $2 \mathrm{D}$ images of biological tissue samples with thicknesses of as much as $25 \mathrm{~mm}$. We demonstrated that the technique was not significantly influenced by speckle decorrelation, that it could be easily implemented, and that it produced better image contrast than the parallel-detection technique.

\section{Experiment}

The experimental setup is shown in Fig. 1, in which a coordinate system is given for reference. The optical axis was along the $X$ axis, and the ultrasonic axis

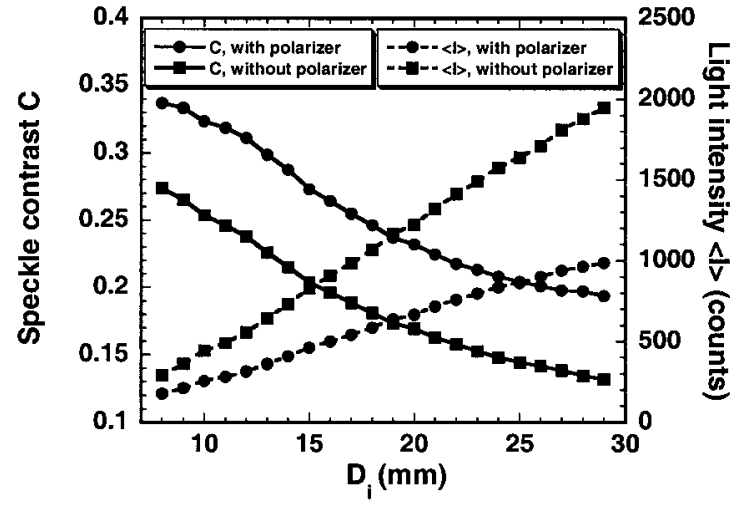

Fig. 2. Variations of measured speckle contrasts and light intensities with the iris aperture $\mathrm{D}_{\mathrm{i}}$. Results with and without a polarizer are compared.

was along the $Z$ axis. A diode laser (56IMS667, 690-nm wavelength; Melles Griot, Inc., Carlsbad, California) was used as the light source. The coherence length of the laser without modulation was more than $1 \mathrm{~m}$. The laser beam incident upon the sample was $\sim 8 \mathrm{~mm}$ in diameter and had a power of $\sim 24 \mathrm{~mW}$. Ultrasonic waves were generated by a focused ultrasonic transducer (Ultran VHP100-1-R38) with a $38-\mathrm{mm}$ focal length in water and a $1-\mathrm{MHz}$ central response frequency. They were coupled with the tissue sample through water, in which the sample was partially immersed. The focal zone of the ultrasonic waves was $\sim 2 \mathrm{~mm}$ in diameter and $\sim 20 \mathrm{~mm}$ in length. The light transmitted through the tissue generated a speckle pattern, which was detected by a 12-bit digital CCD camera of $256 \times 256$ pixels (CA-D1-0256T, Dalsa, Inc., Waterloo, Canada). The exposure time of the CCD camera was set to ensure that sufficient photons were collected. An iris was placed behind the sample to control the average speckle size $D_{s}$ on the CCD surface, with the relation $D_{s} \propto \lambda L / D_{i}$, where $\lambda$ was the light wavelength; $L$ was the distance between the iris and the CCD surface; and $D_{i}$ was the iris aperture. An analyzer was applied to enhance the speckle contrast.

The speckle contrast of a speckle pattern is defined as the ratio $\sigma_{I} /\langle I\rangle$, where $\sigma_{I}$ is the standard deviation of intensity in the pattern and $\langle I\rangle$ is the mean of the intensity. In the experiments, 50 measurements were averaged to improve the SNR. To obtain 2D images, we mechanically scanned the sample with a translation stage along the $X$ and $Y$ directions.

\section{Results and Discussion}

To obtain high speckle contrasts, we adjusted the iris aperture to match the speckle size to the CCD pixel size, which was $16 \mu \mathrm{m}$. It was found that there was a trade-off between the speckle contrast and the light intensity detected by the CCD camera. Figure 2 shows the variations in the speckle contrast and the light intensity with the size of the iris aperture. Results with and without a polarizer are both shown in the figure. It can be seen that with a decrease in the 

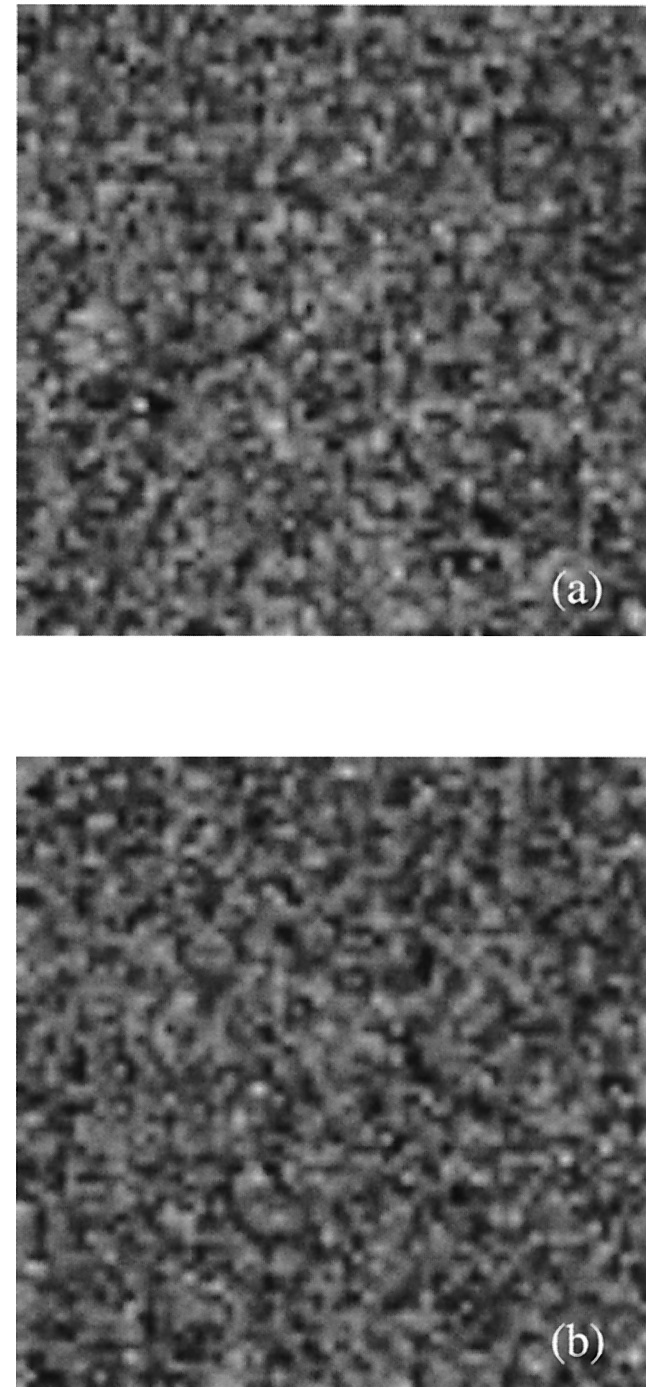

Fig. 3. Speckle patterns measured with the CCD camera. Without ultrasonic modulation, speckle contrast is 0.146 . With ultrasonic modulation, speckle contrast is 0.138 .

aperture (namely, with an increase of the speckle size), the speckle contrast increased, whereas the light intensity decreased. With a polarizer, a higher speckle contrast could be achieved, but the light intensity was much lower. Taking into account that at low intensities the SNR in the detection was low because of the influences of the shot noise and the dark-current noise, we conducted most measurements without a polarizer and with larger iris apertures to keep sufficient SNRs. The speckle contrasts measured in the experiments therefore were relatively low, $\sim 0.14$.

Figure 3 shows two speckle patterns measured when the ultrasound was and was not applied to a chicken tissue sample. Although the change was not visually distinct in Fig. 3(b), the calculation of the speckle contrast showed that the speckle contrast was lower when the ultrasound was applied. We studied the variation of speckle contrast with ultrasound intensity by varying the input power of the

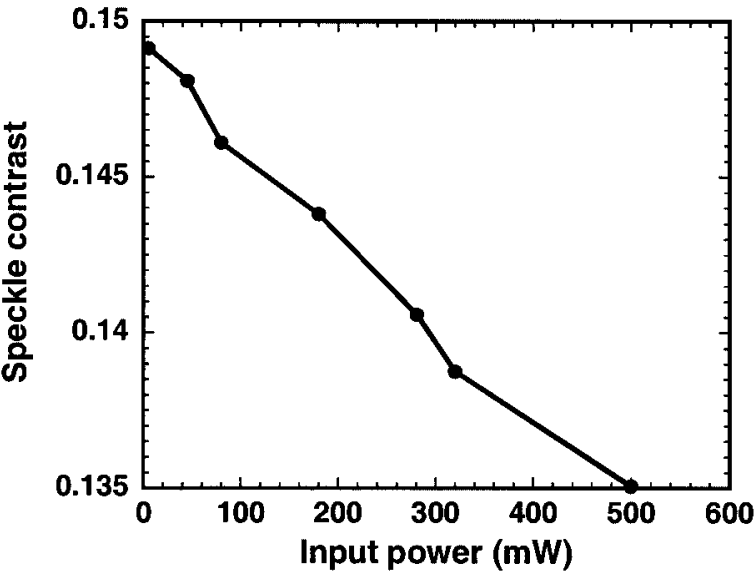

Fig. 4. Variation of speckle contrasts with the input power of the transducer.

transducer. The result is shown in Fig. 4. With increasing power, the speckle contrast decreased rapidly, showing a nearly linear dependence on the input power. We monitored the stability of speckle contrast and found that the stability was more than $99 \%$ within 20 min under a stable condition. So, speckle contrast, which is subject to ultrasound influence, can be used to reflect the ultrasonic modulation of scattered light; that is, it can be used for ultrasoundmodulated optical tomography. In the experiments, we generated efficient ultrasonic modulation with ultrasound pressures within the safety limit for biological tissues by applying an input power of $\sim 280 \mathrm{~mW}$, which generated ultrasonic pressures of $\sim 10^{5} \mathrm{~Pa}$ at the focal spot.

The tissue samples used in the experiments were skinless chicken breast (muscle) tissues, with thicknesses ranging from 15 to $25 \mathrm{~mm}$. Two kinds of optically absorbing objects were used: soft rubber and chicken gizzards. The soft rubber has good acoustic coupling with tissue and has little acoustic absorption. The samples with gizzard objects were all-biological-tissue samples. An ultrasound examination of such a sample with a clinical ultrasonic imager showed that the gizzard had almost the same acoustic impedance as the chicken breast tissue. We measured the optical coefficients of the chicken breast tissue and the gizzard objects with our oblique-incidence reflectometry. ${ }^{21}$ The gizzard showed nearly two times as much absorption but almost identical scattering as the chicken breast tissue.

In the experiments, we measured the speckle contrasts under two conditions: with and without ultrasonic modulation. Figure 5 shows a comparison of three 1D images. In two of them, speckle contrasts were taken as imaging signals, which were measured with and without ultrasonic modulation. In the third one, differences of the speckle contrasts were taken as imaging signals. The sample was a 15-mm-thick chicken breast tissue, in which two rubber objects were buried. The objects are not seen in the two images obtained directly from the speckle contrasts, but they are clearly seen in the image pro- 


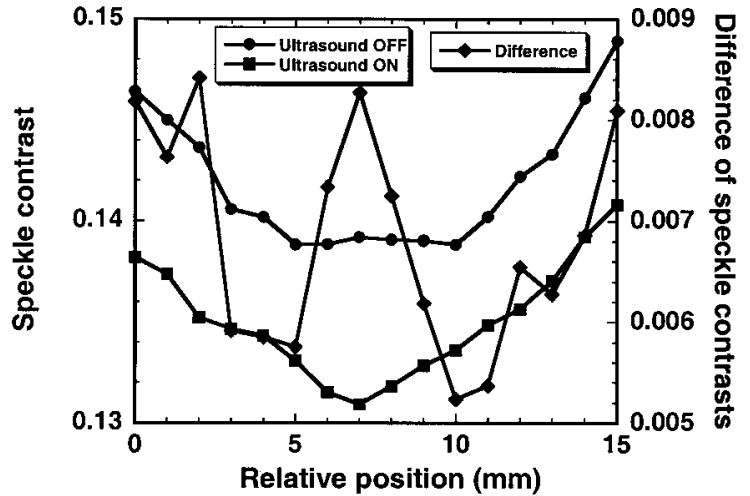

Fig. 5. Comparison of 1D images of two rubber objects that were obtained from speckle contrasts measured with and without ultrasonic modulation and from the difference of the speckle contrasts.

duced by the difference between the two. Subtraction between the speckle contrasts eliminated background contributions and thus provided good imaging signals.

Figure 6(a) shows a 2D image obtained from a 25mm-thick chicken breast tissue sample containing two rubber objects that were buried in the middle plane along the $X$ axis and separated away along the $Y$ axis by approximately $6 \mathrm{~mm}$. The sizes of the two objects were approximately $1.9 \mathrm{~mm} \times 3.1 \mathrm{~mm} \times 10$ $\mathrm{mm}$ and $2.1 \mathrm{~mm} \times 3.1 \mathrm{~mm} \times 10 \mathrm{~mm}$, along the $X, Y$, and $Z$ axes. In the image, the objects are clearly seen. For comparison, the mean intensity $\langle I\rangle$ of the
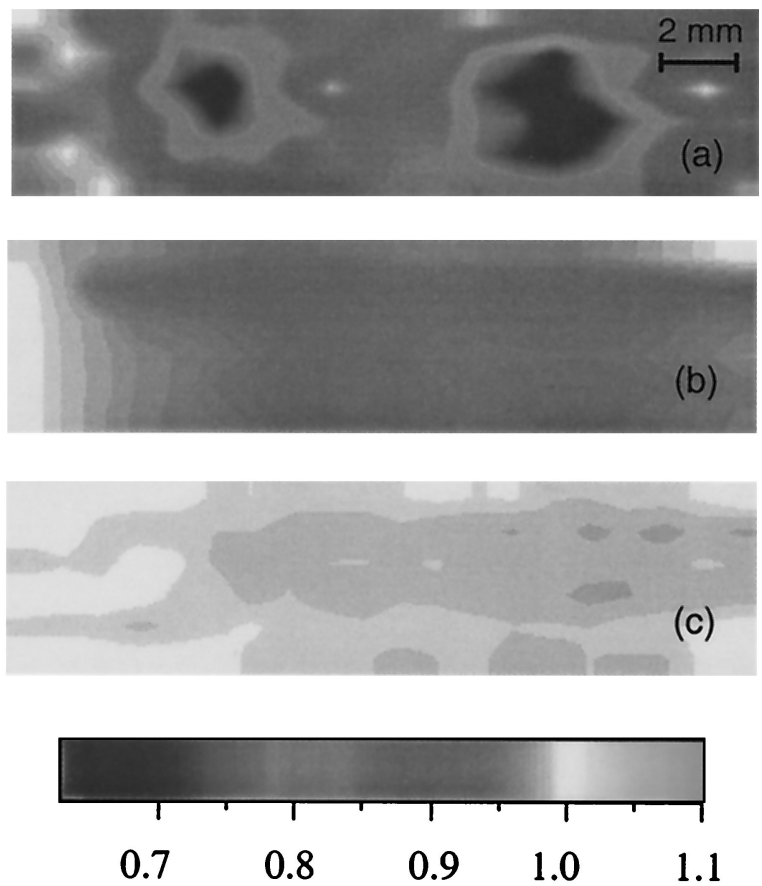

Fig. 6. Two-dimensional images of two rubber objects buried in a 25-mm-thick chicken breast tissue sample: (a) image obtained from the difference of speckle contrasts measured with and without ultrasonic modulation, (b) image obtained from the mean intensity of the speckle pattern, (c) image obtained from the speckle contrast measured without ultrasonic modulation.
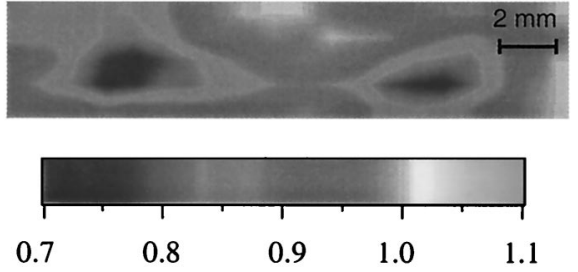

(a)

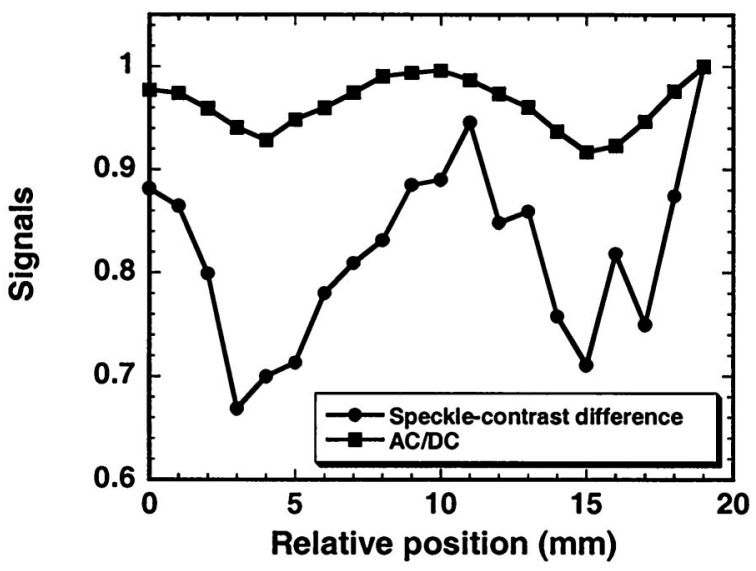

(b)

Fig. 7. (a) Two-dimensional images of two gizzard objects buried in a 17-mm-thick chicken breast tissue sample. (b) Comparison of 1D images obtained with speckle-contrast and parallel detection. The signal obtained in the parallel detection is represented by ac/dc.

speckle pattern and the speckle contrast obtained without ultrasonic modulation were also used as imaging signals, and the images are shown in Figs. 6(b) and 6(c), respectively. The objects are not visible in these two cases. Figure 6(b) shows that the detected light intensity could not directly generate an image of the buried objects. Figure 6(c) shows a result similar to that in Fig. 5, indicating that the speckle contrasts obtained without ultrasonic modulation could not generate images of buried objects. In this case, the imaging was purely optical imaging, just like that taken by Thompson et al. ${ }^{15}$ whereas the objects we used were absorbing media instead of scattering media. Figure 6 shows that ultrasonic modulation in speckle-contrast imaging acts similarly to the way it acts in other modalities of ultrasound-modulated optical tomography-it provides spatial resolution.

Figure 7(a) shows a 2D image of a $17-\mathrm{mm}$-thick sample with two buried gizzard objects. The original sizes of the two objects were approximately 2 $\mathrm{mm} \times 3 \mathrm{~mm} \times 8 \mathrm{~mm}$ and $2 \mathrm{~mm} \times 3 \mathrm{~mm} \times 10 \mathrm{~mm}$. The sizes were changed after the sample was pressed with two parallel planes to maintain a uniform thickness in the $X$ direction. The objects are sharply visible in the image. With the same sample, we performed imaging using parallel detection and compared the results. Figure $7(\mathrm{~b})$ presents the $1 \mathrm{D}$ images obtained by use of either parallel ${ }^{7}$ or of speckle- 
contrast detection. The two experiments were conducted with the same laser and ultrasound intensities. In the figure, ac/dc represents the imaging signal in parallel detection. Both the ac/dc and the speckle-contrast difference are normalized. It can be seen that the image contrast obtained with the present technique is much higher; in fact, it is approximately three times as high as that obtained with parallel detection. It is also shown that the signal fluctuation was more significant in the speckle-contrast detection. One possible reason for the observed fluctuation is that the number of measurements was not sufficient. In the experiments, we obtained one contrast value from each measurement. The average from more than 50 measurements might not have been sufficient to reduce the fluctuation.

Our proposed detection technique utilizes the influence of ultrasonic modulation on speckle-intensity contrasts. When ultrasound interacts with laser light passing through tissues, it modulates the light 13,14 through the modulation of displacements of scatterers and the index of refraction. The modulation causes the speckle intensity detected by a certain pixel of the CCD camera to fluctuate at the ultrasound frequency as well as at its harmonics. We derived the relation between the speckle contrast and the ultrasonic modulation depth. The light intensity on a pixel of the CCD camera can be expressed

$$
I=I_{b}+I_{m}+2\left(I_{b} I_{m}\right)^{1 / 2} \cos \left(\omega_{a} t+\Delta \phi\right),
$$

where $I_{b}$ and $I_{m}$ are the intensities of unmodulated and modulated light, respectively; $\omega_{a}$ is the ultrasonic frequency; and $\Delta \phi$ is the phase difference between the unmodulated and the modulated light. Because the CCD camera runs at low frequencies, the intensity recorded by the camera is time averaged, which is given by

$$
\bar{I}=I_{b}+I_{m}+\frac{1}{T} \int_{0}^{T} 2\left(I_{b} I_{m}\right)^{1 / 2} \cos \left(\omega_{a} t+\Delta \phi\right) \mathrm{d} t
$$

where $T$ is the CCD exposure time. Averaging Eq. (2) over the speckle field, with the statistical property of the speckle pattern, ${ }^{22}$ i.e., $\langle\sin (\Delta \phi)\rangle=0$, we obtain spatially averaged intensities:

$$
\begin{aligned}
\langle\bar{I}\rangle & =\left\langle I_{b}\right\rangle+\left\langle I_{m}\right\rangle \\
\left\langle\bar{I}^{2}\right\rangle & =\left\langle I_{b}{ }^{2}\right\rangle+\left\langle I_{m}{ }^{2}\right\rangle+2\left\langle I_{b} I_{m}\right\rangle+\left(\frac{2}{T \omega_{a}}\right)^{2}\left\langle I_{b} I_{m}\right\rangle,
\end{aligned}
$$

where \langle\rangle represents the spatial-average operation. The variance of the intensity $\sigma^{2}$ and the speckle contrast $C$ are thus obtained:

$$
\begin{aligned}
\sigma^{2}= & \left\langle\bar{I}^{2}\right\rangle-\langle\bar{I}\rangle^{2}=\left\langle I_{b}{ }^{2}\right\rangle-\left\langle I_{b}\right\rangle^{2}+\left\langle I_{m}{ }^{2}\right\rangle-\left\langle I_{m}\right\rangle^{2} \\
& +\left(\frac{2}{T \omega_{a}}\right)^{2}\left\langle I_{b} I_{m}\right\rangle, \\
C= & \frac{\sigma}{\langle\bar{I}\rangle}=\frac{\left[C_{b}{ }^{2}+C_{m}{ }^{2} M^{2}+\left(2 / T \omega_{a}\right)^{2} M\right]^{1 / 2}}{1+M},
\end{aligned}
$$

where,

$$
\begin{aligned}
C_{b} & =\frac{\left(\left\langle I_{b}{ }^{2}\right\rangle-\left\langle I_{b}\right\rangle^{2}\right)^{1 / 2}}{\left\langle I_{b}\right\rangle}, \\
C_{m} & =\frac{\left(\left\langle I_{m}{ }^{2}\right\rangle-\left\langle I_{m}\right\rangle^{2}\right)^{1 / 2}}{\left\langle I_{m}\right\rangle}, \\
M & =\frac{\left\langle I_{m}\right\rangle}{\left\langle I_{b}\right\rangle} .
\end{aligned}
$$

$M$ is the ultrasonic modulation depth. $C_{b}$ and $C_{m}$ can be considered contrasts of two speckle fields with mean intensities of $\left\langle I_{b}\right\rangle$ and $\left\langle I_{m}\right\rangle$, respectively. Because we used 1-MHz ultrasound and $\sim 20 \mathrm{~ms}$ CCD exposure time in the experiments, i.e., $1 / T \omega_{a} \ll 1$, and the modulation depth was much less than unity, the second and the third terms in the numerator in Eq. (6) are small and thus can be ignored. The speckle contrast can be expressed

$$
C \approx \frac{C_{b}}{1+M}
$$

which shows that the speckle contrast decreases approximately linearly with the increase of the ultrasonic modulation depth.

According to ultrasonic modulation analysis, ${ }^{13}$ one can determine that the modulation depth is proportional to the square of the ultrasonic amplitude; namely, the modulation depth is proportional to the ultrasonic or the electric power input to the transducer. By combining this relation and approximation (10) one can explain the approximately linear decrease in the speckle contrast with the power input in Fig. 4.

If a portion of the incident light is absorbed by objects located inside the ultrasonic column, the influences of ultrasound on the light and, as a consequence, on the speckle contrast are weakened; that is, the speckle contrast decreases less. Therefore the variation in speckle contrast can provide information for imaging. Further, the differences in those speckle contrasts that are obtained with and without ultrasonic modulation provide image signals directly. By scanning the sample, one can obtain images having spatial resolution determined by the focal spot of the ultrasound. Purely optical imaging based on speckle contrasts was successfully demonstrated by Thompson et al. ${ }^{15}$ in their experiments on imaging a scattering object. In our experiments, however, we found that purely optical imaging was not efficient for detecting absorbing objects. Our results show that with ultrasonic modulation, the speckle contrast can be used for imaging absorbing objects. In the experiments, we used objects with strong optical absorption properties, which gave satisfactory image contrasts. If the objects had been less absorbent, the image contrast would have been lower. Because the measured signal, i.e., the speckle-contrast difference, is small, imaging less-absorbent objects is more challenging. In future studies, an objective will be to 
improve the detection method so that low-contrast objects are more efficiently imaged.

When using parallel detection, ${ }^{6-9,12}$ one should be careful with the speckle decorrelation caused by the Brownian motion of scatterers, which may occur during the four-acquisition measurement period. A correlation time of $400 \mathrm{~ms}$ was measured in our previous experiments with 12 -mm-thick in vitro chicken tissues, ${ }^{7}$ and the correlation time was found to decrease with the increase of tissue thickness, as was expected. In parallel detection, the total dataacquisition time must be shorter than the correlation time. However, sufficient exposure is needed in the case of low laser power or thick samples. With our speckle-contrast detection method, acquisitions with and without ultrasonic modulation at a location can be nearly independent, and the speckle decorrelation can be avoided if each acquisition time is short enough. Compared with parallel detection, our technique requires fewer components and is easy to conduct.

\section{Conclusion}

Using the speckle-contrast mechanism in ultrasoundmodulated tomography, we obtained 2D images of biological-tissue samples of as much as $25 \mathrm{~mm}$ thick by use of a low laser intensity of $\sim 24 \mathrm{~mW}$. The technique is superior to speckle-contrast-based, purely optical imaging because its use of ultrasonic resolution allows it to discriminate absorbing objects. Comparison showed that images obtained with this technique had better contrast than those obtained with parallel detection and that speckle decorrelation was less significant in the detection. In addition, the ultrasound-modulated tomography technique was simple to setup. It combines speckle-contrast detection with ultrasonic modulation, thus providing an efficient method for ultrasound-modulated tomography of biological tissues.

This project was sponsored in part by the National Institutes of Health grant R01 CA71980; the National Science Foundation grant BES-9734491; and the Texas Higher Education Coordinating Board grant 000512-0123-1999.

\section{References}

1. F. A. Marks, H. W. Tomlinson, and G. W. Brooksby, "Comprehensive approach to breast cancer detection using light: photon localization by ultrasonic modulation and tissue characterization by spectral discrimination," in Photon Migration and Imaging in Random Media and Tissue, B. Chance and R. R. Alfano, eds., Proc. SPIE 1888, 500-510 (1993).

2. L.-H. Wang, S. L. Jacques, and X. Zhao, "Continuous-wave ultrasonic modulation of scattered laser light to image objects in turbid media," Opt. Lett. 20, 629-631 (1995).

3. L.-H. Wang and X. Zhao, "Ultrasound-modulated optical to- mography of absorbing objects buried in dense tissuesimulating turbid media," Appl. Opt. 36, 7277-7282 (1997).

4. W. Leutz and G. Maret, "Ultrasonic modulation of multiply scattered light," Phys. B 204, 14-19 (1995).

5. L.-H. Wang and G. Ku, "Frequency-swept ultrasoundmodulated optical tomography of scattering media," Opt. Lett. 23, 975-977 (1998).

6. S. Leveque, A. C. Boccara, M. Lebec, and H. Saint-Jalmes, "Ultrasonic tagging of photon paths in scattering media: parallel speckle modulation processing," Opt. Lett. 24, 181-183 (1999).

7. G. Yao and L.-H. Wang, "Theoretical and experimental studies of ultrasound-modulated optical tomography in biological tissue," Appl. Opt. 39, 659-664 (2000).

8. G. Yao, S. Jiao and L.-H. Wang, "Frequency-swept ultrasoundmodulated optical tomography in biological tissue by use of parallel detection," Opt. Lett. 25, 734-736 (2000).

9. S. Leveque-Fort, "Three-dimensional acousto-optic imaging in biological tissues with parallel signal processing," Appl. Opt. 40, 1029-1036 (2000).

10. A. Lev, Z. Kotler, and B. G. Sfez, "Ultrasound tagged light imaging in turbid media in a reflectance geometry," Opt. Lett. 25, 378-380 (2000).

11. E. Granot, A. Lev, Z. Kotler, and B. G. Sfez, "Detection of inhomogeneities with ultrasound tagging of light," J. Opt. Soc. Am. A 18, 1962-1967 (2001).

12. S. Leveque-Fort, J. Selb, L. Pottier, and A. C. Boccara, "In situ local tissue characterization and imaging by backscattering acousto-optic imaging," Opt. Commun. 196, 127-131 (2001).

13. L.-H. V. Wang, "Mechanisms of ultrasonic modulation of multiply scattered coherent light: an analytic model," Phys. Rev. Lett. 87, (043093) 1-4 (2001).

14. L.-H. V. Wang, "Mechanisms of ultrasonic modulation of multiply scattered coherent light: a Monte Carlo model," Opt. Lett. 26, 1191-1193 (2001).

15. C. A. Thompson, K. J. Webb, and A. M. Weiner, "Imaging in scattering media by use of laser speckle," J. Opt. Soc. Am. A 14, 2269-2277 (1997).

16. J. D. McKinney, M. A. Webster, K. J. Webb, and A. M. Weiner, "Characterization and imaging in optically scattering media by use of laser speckle and a variable-coherence source," Opt. Lett. 25, 4-6 (2000).

17. P. Naulleau, D. Dilworth, E. Leith, and J. Lopez, "Detection of moving objects embedded within scattering media by use of speckle methods," Opt. Lett. 20, 498-500 (1995).

18. G. Parry, "Some effects of temporal coherence on the first order statistics of speckle," Opt. Acta 21, 763-772 (1974).

19. D. A. Zimnyakov, V. V. Tuchin, and S. R. Utts, "A study of statistical properties of partially developed speckle fields as applied to the diagnostics of structural changes in human skin," Opt. Spectrosc. 76, 838-844 (1994).

20. S. L. Jacques and S. J. Kirkpatrick, "Acoustically modulated speckle imaging of biological tissues," Opt. Lett. 23, 879-881 (1998).

21. G. Marquez and L.-H. Wang, "White light oblique incidence reflectometer for measuring absorption and reduced scattering spectra of tissue-like turbid media," Opt. Express 1, 454-460 (1997).

22. J. W. Goodman, "Statistics properties of laser speckle patterns," in Laser Speckle and Related Phenomenon, J. C. Dainty, ed. (Springer-Verlag, Berlin, 1975), pp. 9-75. 